Pacific Journal of Mathematic 


\title{
MAXIMAL SUBGROUPS AND CHIEF FACTORS OF CERTAIN GENERALIZED SOLUBLE GROUPS
}

\author{
Richard E. Phillips, Derek J. S. Robinson \\ AND JAMES E. ROSEblade
}

It is shown by means of a generalization of a result of R. Baer and D. H. McLain that if $G$ is a locally polycyclic group and if the chief factors of every finitely generated subgroup of $G$ have finite rank at most equal to $r$, then every maximal subgroup of $G$ has index dividing the $r$ th power of some prime. This answers a question about locally supersoluble groups raised by the first author. In addition, examples are furnished to show that neither of the properties "all chief factors are finite" and "all maximal subgroups have finite index" implies the other.

1. Both R. Baer [1, p. 419] and D. H. McLain [9] have given proofs of the fact that maximal subgroups of locally nilpotent groups are normal. An easy generalization of this, which seems hitherto to have escaped notice, is

THEOREM A. Let $K$ be a normal locally nilpotent subgroup of a locally Noetherian group $G$. If $H$ is a maximal subgroup of $G$, then $H \cap K$ is normal in $G$.

The proof is similar to McLain's proof of the special case when $K=G$. Suppose $H \cap K$ is not normal in $G$. Then there exists $x$ in $G$ such that $(H \cap K)^{x} \not \subset H \cap K$. Since $K$ is normal in $G$, it follows that $(H \cap K)^{x}$ is not contained in $H$. Since $H$ is maximal, $G=\left\langle H,(H \cap K)^{x}\right\rangle$. Thus, there exist finitely generated subgroups $P$ of $H$ and $Q$ of $H \cap K$ such that $x \in R=\left\langle P, Q^{x}\right\rangle$. Letting $Q_{1}=Q^{R}$, we deduce that $R=P\left(Q^{x}\right)^{R}=P Q^{R}=P Q_{1}$. Therefore

$$
Q_{1}=\left(Q^{P}\right)^{Q_{1}} \text {. }
$$

Since $R$ is finitely generated, it is Noetherian. Thus its subgroup $Q_{1}$ is finitely generated. Since $Q_{1}$ is contained in $K$ and $K$ is locally nilpotent, $Q_{1}$ is nilpotent. Thus $Q_{1}=\left(Q^{P}\right)^{Q_{1}}$ implies that $Q_{1}=Q^{P}$, which in turn implies that $R=\langle P, Q\rangle \leqq H$. Since $x$ belongs to $R$ but not to $H$, we have a contradiction and the theorem is proved.

As a simple consequence of Theorem $\mathrm{A}$ we have

CoRollary 1. If $G$ is a locally Noetherian, radical group and 
$H$ is a maximal subgroup of $G$, there exists a chief factor $U / V$ of $G$ such that $H U=G$ and $H \cap U=V$.

Here we are using the term radical in the sense of Plotkin [12]; a group $G$ is radical if every nontrivial homomorphic image has a nontrivial locally nilpotent normal subgroup.

To prove the corollary suppose that $H$ is a maximal subgroup of $G$ and let $V$ be the core of $H$ in $G$. Since $G$ is a radical group, there is a nontrivial locally nilpotent normal subgroup $U / V$ of $G / V$. Application of Theorem A to $G / V$ shows that $H \cap U=V$. Suppose that $L \triangleleft G$ and $V<L \leqq U$. Then certainly $L \not \subset H$, so that $G=H L$. Hence $U=U \cap(H L)=V L=L$. It follows that $U / V$ is a chief factor of $G$ and that $G=H U$.

We remark that the index of $H$ in $G$ equals the order of $U / V$. Therefore, if all the chief factors of $G$ are finite, then so are the indices of all the maximal subgroups of $G$. This allows us to prove

CoRollary 2. Suppose that $G$ is a locally polycyclic group. If the chief factors of every finitely generated subgroup of $G$ all have rank at most $r$, then every maximal subgroup of $G$ has index dividing the $r$-th power of some prime.

Since chief factors of supersoluble groups have prime order, it follows from this that any maximal subgroup of a locally supersoluble group has prime index. This answers a question raised by $\mathrm{R}$. E. Phillips in [11; p. 350].

To prove this corollary it is enough by the first corollary and a theorem of McLain [10; p. 104] to show that the group $G$ is radical. This can be done by using the theorem of Zassenhaus [13; p. 294] which asserts the existence of an integer $r^{*}$, depending only on $r$, such that any soluble linear group of degree $r$ has derived length at most $r^{*}$.

Suppose that $X$ is any finitely generated subgroup of $G$-so that $X$ is polycyclic-and let $Y=X^{\left(r^{*}\right)}$, the $r^{*}$ th term of the derived series of $X$. If $Y^{n}$ denotes the subgroup of $Y$ generated by all $n$th powers of elements of $Y$, then $Y / Y^{n}$ is finite and consequently has a series the factors of which are chief factors of $X$. All these must be centralized by $Y$, so $Y / Y^{n}$ is nilpotent and hence every finite homomorphic image of $Y$ is nilpotent. We deduce from a theorem of $\mathrm{K}$. A. Hirsch [6; p. 190] that $Y$ is nilpotent.

It follows easily that $G^{\left(r^{*}\right)}$ is locally nilpotent and therefore that $G$ is a radical group.

2. The question arises whether there exist generalized soluble 
groups which have maximal subgroups of infinite index but all of whose chief factors are finite. We shall show that such groups do exist by considering a cyclic extension of one of the groups discussed by McLain in [7].

Let $F$ be a field with a prime number of elements and suppose that $V$ is a vector space over $F$ with basis elements $v_{n}, n=0, \pm 1$, $\pm 2, \cdots$, . For integers $m<n$ we shall write $\tau_{m n}$ for the linear transformation of $V$ determined by

$$
v_{m} \tau_{m, n}=v_{m}+v_{n} \quad \text { and } \quad v_{r} \tau_{m n}=v_{r} \text { if } r \neq m .
$$

The group $M$ generated by all the $\tau_{m, n}$ is the McLain group in question. The linear transformation $t$ of $V$ which sends each $v_{n}$ onto $v_{n+1}$ transforms $\tau_{m, n}$ into $\tau_{m+1, n+1}$ and therefore normalizes $M$. We define $G$ to be the group generated by $M$ and $t$. We shall prove

THEOREM B. Every chief factor of $G$ is finite and yet $G$ has a maximal subgroup of infinite index.

We show first that the chief factors of $G$ are all finite. Since those of $G / M$ obviously are, it suffices to consider chief factors $U / V$ of $G$ with $U \leqq M$. By an argument of McLain [8], every nontrivial normal subgroup of $M$ contains one of the generators $\tau_{i, j}$. If the normal subgroup is invariant under $\langle t\rangle$, then it must clearly contain one of the subgroups

$$
\gamma_{n}(M)=\left\langle\tau_{i, i+n} \mid i=0, \pm 1, \pm 2, \cdots\right\rangle
$$

of $M$. However it is well known and easy to see that $\gamma_{n}(M)$ is the $n$th term of the lower central series of $M$ and also that $\bigcap_{n} \gamma_{n}(M)$ is trivial. This shows that $V$ is nontrivial and therefore $M / V$ is nilpotent. It follows that $U / V$ is a central factor of $M$ and consequently an irreducible representation space for $\langle t\rangle$ over $F$. By Theorem 3.1 of P. Hall [5] or a simple direct argument we deduce that $U / V$ is finite, as required.

We turn now to the existence of maximal subgroups of $G$ which are of infinite index. It will be enough to show that the subgroup

$$
K=\left\langle\tau_{0,1} \tau_{0,2}, t\right\rangle
$$

does not contain the element $\tau_{0,1}$. For suppose this has been done. It is clear that $G$ is generated by $\tau_{01}$ and $t$. Let $H$ be a subgroup of $G$ containing $K$ which is maximal with respect to excluding $\tau_{0,1}$. Clearly $H$ is a maximal subgroup of $G$ and, by (1), $G=H \gamma_{2}(M)$. Therefore, by a well-known argument, $G=H \gamma_{n}(M)$ for each positive integer $n$. However $M$ is locally nilpotent, so a subgroup of finite 
index must contain a term of the lower central series of $M$. Therefore $[M: M \cap H]=[G: H]$ is infinite.

Now $K=N\langle t\rangle$, where $N=\left(\tau_{0,1} \tau_{02}\right)^{\langle t\rangle}=M \cap K$.

We must show that

$$
\tau_{0,1} \notin N
$$

and in order to achieve this we shall write

and

$$
M^{-}=\left\langle\tau_{m, n} \mid m<0\right\rangle
$$

$$
M^{+}=\left\langle\tau_{m, n} \mid m \geqq 0\right\rangle \text {. }
$$

It is easy to see that $M^{-}$is a normal subgroup of $M$ and that

$$
\begin{aligned}
M & =M^{+} M^{-}, \\
1 & =M^{+} \cap M^{-} .
\end{aligned}
$$

We define

and

$$
\xi_{n}=\tau_{0 n} \tau_{0, n+1}, \quad(n \geqq 1)
$$

$$
\eta_{m}=\tau_{m, m+1} \tau_{m, m+2}, \quad(m=0, \pm 1, \pm 2, \cdots),
$$

and let $Z=\left\langle\xi_{n}, \eta_{n} \mid n \geqq 1\right\rangle$.

It is clear that $Z \leqq M^{+}$, and that

$$
N=\left\langle\xi_{1}, \eta_{n} \mid n= \pm 1, \pm 2, \cdots\right\rangle \leqq Z M^{-} .
$$

(3), (4) and (5) together show that (2) will follow from showing that

$$
\tau_{0.1} \notin Z \text {. }
$$

Let $X=\left\langle\xi_{n} \mid n \geqq 1\right\rangle$ and $Y=\left\langle\eta_{n} \mid n \geqq 1\right\rangle$. For $n \geqq 1$, the element $\xi_{n}$ commutes with every $\eta_{r}, r \geqq 1$, except $\eta_{n}$ and $\eta_{n+1}$. Moreover, $\left[\xi_{n}, \eta_{n}\right]=\xi_{n+1}$ and $\left[\xi_{n}, \eta_{n+1}\right]=\xi_{n+2}$. This shows that $Y$ normalizes $X$. Therefore $Z=X Y$. Suppose $\tau_{01} \in Z$; then $\tau_{0,1}=\xi \eta$ for some $\xi \in X$ and some $\eta \in Y$. But then $\xi^{-1} \tau_{0,1}=\eta$ and this element fixes $v_{n}$ for $n \neq 0$ since both $\xi$ and $\tau_{0.1}$ do, and also fixes $v_{0}$ because $\eta$ does. Hence $\eta=1$ and $\xi=\tau_{0,1}$ belongs to $X$. However, the elements $\tau_{0,1}, \tau_{0,2}, \cdots$, $\tau_{0, n} \cdots$ form a basis of the elementary abelian group which they generate, and therefore $\tau_{0,1}$ cannot belong to $X$. This establishes (6).

We note that this group $G$ is a 2-generator radical group. Since $M$ is generated by its abelian normal subgroups $\left\langle L_{n, m}^{M}\right\rangle$, the group $G$ is even subsoluble in the sense of Baer [2: p. 421]. Moreover, the group $G$ satisfies Max- $n$, the maximal condition for normal subgroups. To see this, we remark that for each $n$ the lower central factor $\gamma_{n}(M) / \gamma_{n+1}(M)$ is isomorphic as an $F\langle t\rangle$-module with the group algebra 
$F\langle t\rangle$ itself and so, according to a theorem of P. Hall [4: p. 429], is a Noetherian module. It follows that each of the groups $G / \gamma_{n}(M)$ satisfies Max- $n$. Since every nontrivial normal subgroup of $G$ must contain some $\gamma_{n}(M)$, it follows that $G$ has Max- $n$.

Thus even with these stringent additional hypotheses the finiteness of all the chief factors of a group does not imply that each maximal subgroup is of finite index. On the other hand, it is easy to prove that a hyperabelian (or $S I^{*_{-}}$) group with finite chief factors has every maximal subgroup of finite index. Hyperabelian groups, of course, form a rather special class of subsoluble groups.

3. We conclude by observing that the same situation prevails in the opposite direction. More precisely we shall establish

THEOREM C. There exists a metabelian group which has no maximal subgroups and yet has infinite chief factors.

For let $H$ be a quasicyclic group of type $C_{p \infty}$ and $K$ a group of type $C_{q \infty}$ where $p$ and $q$ are distinct primes. The group which will demonstrate Theorem $\mathrm{C}$ is the restricted standard wreath product $W=H \imath K$. Let $B=H^{W}$, the base group of $W$. Since $W / B \cong K$, no maximal subgroup of $W$ can contain $B$. Thus if $L$ is a maximal subgroup of $W$, it follows that $W=B L$ and $B / B \cap L$ is a chief factor of $W$. But $B$ is a radicable abelian p-group, so this is impossible. Hence $W$ has no maximal subgroups.

On the other hand $W$ does have infinite chief factors: for if $x$ is an element of $H$ with order $p$, then $\langle x\rangle^{W}$ is isomorphic as an $F K$ module with the group algebra $F K$ where $F$ is a field with $p$ elements, and it has been shown by Čarin [3] that $K$ has an infinite dimensional irreducible representation over $F$.

\section{REFERENCES}

1. R. Baer, Nilpotent groups and their generalizations, Trans. Amer. Math. Soc., 47 (1940), 393-434.

2. Wil-Gruppen, Math. Zeit., 62 (1955), 402-437.

3. V. S. Čarin, $A$ remark on the minimal condition for subgroups, Dokl. Akad. Nauk SSSR (N.S) 66 (1949), 575-576 (Russian).

4. P. Hall, Finiteness conditions for soluble groups, Proc. London Math. Soc., (3) 4 (1954), 419-436.

5. — On the finiteness of certain soluble groups, Proc. London Math. Soc., (3)

9 (1959), 595-622.

6. K. A. Hirsch, On infinite soluble groups III, Proc. London Math. Soc., (2), 49 (1946), 184-194. 
7. D. H. McLain, A Class of Locally Nilpotent Groups, Ph. D. Dissertation, Cambridge University, Cambridge, England, (1956).

8. — A characteristically simple group, Proc. Cambridge Philos. Soc., 50 (1954), 641-642.

9. - On locally nilpotent groups, Proc. Cambridge Philos. Soc., 52 (1956), 5-11. 10. - Finiteness conditions in locally soluble groups, J. London Math. Soc., 34 (1959), 101-107.

11. R. E. Phillips, F-systems in infinite groups, Arch. Math., 20 (1969), 345-355.

12. B. I. Plotkin, Radical groups, Mat. Sbornik 9 (1954), 181-186=Amer. Math. Soc. Translations (2) 17 (1961), 9-28.

13. H. Zassenhaus, Beweis eines Satzes ïber diskrete Gruppen, Abh. Math. Sem. Univ. Hamburg 12 (1938), 289-312.

Received September 21, 1970. The third author wishes to thank the Mathematics Department at Michigan State University for splendid hospitality whilst this paper was being prepared. The second author acknowledges support from the National Science Foundation.

Michigan State University, East Lansing, Michigan

UNiversity of Illinois, Urbana, IllinoIS

AND

Jesus College, Cambridge, England 


\section{PACIFIC JOURNAL OF MATHEMATICS}

\section{EDITORS}

\author{
H. SAMElson \\ Stanford University \\ Stanford, California 94305 \\ C. R. HOBBY \\ University of Washington \\ Seattle, Washington 98105
}

J. DugundjI

Department of Mathematics University of Southern California Los Angeles, California 90007

RICHARD ARENS

University of California

Los Angeles, California 90024

\section{ASSOCIATE EDITORS}
E. F. BECKENBACH
B. H. NeUmanN
F. WOLE
K. YOSHIDA

\section{SUPPORTING INSTITUTIONS}

\author{
UNIVERSITY OF BRITISH COLUMBIA \\ CALIFORNIA INSTITUTE OF TECHNOLOGY \\ UNIVERSITY OF CALIFORNIA \\ MONTANA STATE UNIVERSITY \\ UNIVERSITY OF NEVADA \\ NEW MEXICO STATE UNIVERSITY \\ OREGON STATE UNIVERSITY \\ UNIVERSITY OF OREGON \\ OSAKA UNIVERSITY \\ UNIVERSITY OF SOUTHERN CALIFORNIA
}

\author{
STANFORD UNIVERSITY \\ UNIVERSITY OF TOKYO \\ UNIVERSITY OF UTAH \\ WASHINGTON STATE UNIVERSITY \\ UNIVERSITY OF WASHINGTON \\ * * * \\ AMERICAN MATHEMATICAL SOCIETY \\ CHEVRON RESEARCH CORPORATION \\ NAVAL WEAPONS CENTER
}

The Supporting Institutions listed above contribute to the cost of publication of this Journal, but they are not owners or publishers and have no responsibility for its content or policies.

Mathematical papers intended for publication in the Pacific Journal of Mathematics should be in typed form or offset-reproduced, (not dittoed), double spaced with large margins. Underline Greek letters in red, German in green, and script in blue. The first paragraph or two must be capable of being used separately as a synopsis of the entire paper. The editorial "we" must not be used in the synopsis, and items of the bibliography should not be cited there unless absolutely necessary, in which case they must be identified by author and Journal, rather than by item number. Manuscripts, in duplicate if possible, may be sent to any one of the four editors. Please classify according to the scheme of Math. Rev. Index to Vol. 39. All other communications to the editors should be addressed to the managing editor, Richard Arens, University of California, Los Angeles, California, 90024.

50 reprints are provided free for each article; additional copies may be obtained at cost in multiples of 50 .

The Pacific Journal of Mathematics is published monthly. Effective with Volume 16 the price per volume (3 numbers) is $\$ 8.00$; single issues, $\$ 3.00$. Special price for current issues to individual faculty members of supporting institutions and to individual members of the American Mathematical Society: $\$ 4.00$ per volume; single issues $\$ 1.50$. Back numbers are available.

Subscriptions, orders for back numbers, and changes of address should be sent to Pacific Journal of Mathematics, 103 Highland Boulevard, Berkeley, California, 94708.

PUBLISHED BY PACIFIC JOURNAL OF MATHEMATICS, A NON-PROFIT CORPORATION

Printed at Kokusai Bunken Insatsusha (International Academic Printing Co., Ltd.), 7-17, Fujimi 2-chome, Chiyoda-ku, Tokyo, Japan. 


\section{Pacific Journal of Mathematics}

\section{Vol. 37, No. $2 \quad$ February, 1971}

Charles Compton Alexander, Semi-developable spaces and quotient images of metric spaces .................................... 277

Ram Prakash Bambah and Alan C. Woods, On a problem of Danzer. . . . . . . . . 295

John A. Beekman and Ralph A. Kallman, Gaussian Markov expectations and related integral equations . ....................................

Frank Michael Cholewinski and Deborah Tepper Haimo, Inversion of the Hankel

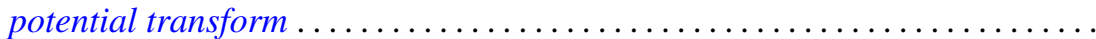

John H. E. Cohn, The diophantine equation

$$
Y(Y+1)(Y+2)(Y+3)=2 X(X+1)(X+2)(X+3) \ldots \ldots \ldots \ldots \ldots
$$

Philip C. Curtis, Jr. and Henrik Stetkaer, A factorization theorem for analytic

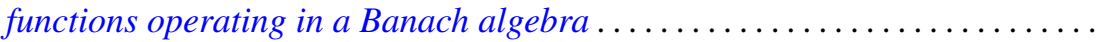

Doyle Otis Cutler and Paul F. Dubois, Generalized final rank for arbitrary limit ordinals

Keith A. Ekblaw, The functions of bounded index as a subspace of a space of entire functions

Dennis Michael Girard, The asymptotic behavior of norms of powers of

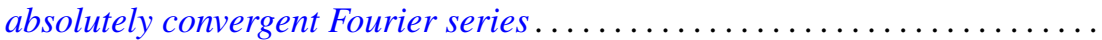

John Gregory, An approximation theory for elliptic quadratic forms on Hilbert spaces: Application to the eigenvalue problem for compact quadratic forms. 383

Paul C. Kainen, Universal coefficient theorems for generalized homology and stable cohomotopy.

Aldo Joram Lazar and James Ronald Retherford, Nuclear spaces, Schauder bases, and Choquet simplexes.

David Lowell Lovelady, Algebraic structure for a set of nonlinear integral operations

John McDonald, Compact convex sets with the equal support property . 429

Forrest Miller, Quasivector topologies

Marion Edward Moore and Arthur Steger, Some results on completability in commutative rings.

A. P. Morse, Taylor's theorem

Richard E. Phillips, Derek J. S. Robinson and James Edward Roseblade, Maximal subgroups and chief factors of certain generalized soluble groups.

Doron Ravdin, On extensions of homeomorphisms to homeomorphisms ...

John William Rosenthal, Relations not determining the structure of $\mathrm{L}$

Prem Lal Sharma, Proximity bases and subbases ........... .

Larry Smith, On ideals in $\Omega_{*}^{u}$. .

Warren R. Wogen, von Neumann algebras generated by operators similar to normal operators

R. Grant Woods, Co-absolutes of remainders of Stone-Čech 